\title{
The function and mechanism of COX-2 in angiogenesis of gastric cancer cells
}

\author{
Liping Yao ${ }^{\dagger}$, Fei Liư ${ }^{\dagger}$ Liu Hong ${ }^{\dagger}$, Li Sun, Shuhui Liang, Kaichun Wư, Daiming Fan ${ }^{*}$
}

\begin{abstract}
Background: Here we aimed to investigate the effect of COX-2 siRNA on proliferation and angiogenesis of gastric cancer cells.

Methods: The gastric cancer cell line SGC7901 was transfected with COX-2 siRNA, then the growth and angiogenesis of cells were detected by in vitro and in vivo assay. Human microarray, RT-PCR and western blot were used to identify differentially expressed angiogenesis-related molecules in cells with decreased expression of COX-2.
\end{abstract}

Results: Down-regulation of COX-2 could significantly inhibit the in vitro and in vivo growth of gastric cancer cells, and suppress the migration and tube formation of human umbilical vein endothelial cells. Totally 23 angiogenesisrelated molecules were found involved in COX-2-induced angiogenesis suppression. The results of RT-PCR and western blot showed that down-regulation of COX-2 might inhibit VEGF, Flt-1, Flk-1/KDR, angiopoietin-1, tie-2, MMP2 and OPN.

Conclusions: COX-2 might mediate tumor angiogenesis and growth, and could be considered as a target for gastric cancer therapy.

\section{Background}

Gastric cancer is the second leading cause of cancer associated death in the world, particularly in Asian countries. The treatment outcome of this common malignancy is still not satisfactory and various chemotherapeutic attempts in an adjuvant setting have failed to improve the survival rate in gastric cancer. Recently, angiogenesis has been found related to hematogenous recurrence and poor prognosis in gastric cancer [1]. Angiogenesis is the growth of new vessels from existing vasculature. A balance of angiogenic and angiostatic growth factors tightly controls physiological angiogenesis. Tipping of this balance towards a pro-angiogenic environment is termed the 'angiogenic switch' and occurs in situations such as tissue hypoxia, inflammation or neoplasia [2].

COX-2, a COX isoenzyme catalyzing the production of prostaglandins, has been observed in most gastric

\footnotetext{
* Correspondence: xiaohuakaichun@126.com; hlhyhj@126.com + Contributed equally

State Key Laboratory of Cancer Biology and Xijing Hospital of Digestive Diseases, Fourth Military Medical University, 15 West Changle Road, Xi'an, 710032, PR China
}

(C) 2011 Yao et al; licensee BioMed Central Ltd. This is an Open Access article distributed under the terms of the Creative Commons

cancer tissues compared with the accompanying normal mucosa. Studies in different cancers have suggested a relationship between COX-2 and increased proangiogenic growth factors, in particular VEGF [3]. COX-2 is thought to promote angiogenesis and so drive the malignant phenotype. Overexpression of COX-2 might contribute to angiogenesis of gastric cancer [4]. However, the potential mechanism underlying the role of COX-2 in angiogenesis remains unclear.

Here we have demonstrated novel observations that COX-2 might play important roles in angiogenesis of gastric cancer through regulation of VEGF, Flt-1, Flk-1/ KDR, angiopoietin-1, tie-2, MMP2 and OPN.

\section{Methods}

\section{Cell culture}

Human gastric cancer cell line SGC7901 was cultivated in Dulbecco's modified Eagle's medium supplemented with $10 \%$ heat-inactivated fetal calf serum, penicillin $(100 \mathrm{U} / \mathrm{ml})$ and streptomycin $(100 \mu \mathrm{g} / \mathrm{ml})$, in a $\mathrm{CO}_{2}$ incubator (Forma Scientific) [5]. Human umbilical vein endothelial cells (HUVEC-12; ATCC, Manassas, VA) were grown in Kaighn's modification of Ham's F12 
medium (ATCC) with $2 \mathrm{mM}$ Lglutamine, $1.5 \mathrm{~g} / \mathrm{l}$ sodium bicarbonate, $0.1 \mathrm{mg} / \mathrm{ml}$ heparin, $0.03 \mathrm{mg} / \mathrm{ml}$ endothelial cell growth supplement and 10\% FBS.

\section{Plasmid construction and transfection}

The siRNA oligos for COX-2 were designed according to previous report. Target sequences were aligned to the human genome database in a BLAST search to ensure that the choosing sequences were not highly homologous with other genes. For oligo-1, S: 5'-tttgcatcgatgtcaccatagaacatctatggtgacatcgatgcttttt-3', AS: $5^{\prime}$-ctagaaaaagcatcgatgtcacc atagatgttctatggtgacatcgatg-3' For annealing to form DNA duplexes, $100 \mu \mathrm{M}$ of each $\mathrm{S}$ and AS oligos was used. The duplexes were diluted and then ligated with mU6pro vector which previously digested by the Bbs I/Xba I restriction enzyme and gel purified at room temperature for $30 \mathrm{~min}$. The products were transformed into DH5 $\alpha$ competent cells. Ampicillin-resistant colonies were chosen, identified by restriction digestion and further confirmed by DNA sequencing.

SGC7901 cells were planted in six-well plates and cultured in drug-free medium. At 90-95\% confluence, cells were washed twice with PBS, grew in $2 \mathrm{ml}$ of DMEM without antibiotics. Using Lipofectamine ${ }^{\mathrm{TM}} 2000$ reagent (Invitrogen, Inc. Carlsbad CA), $2 \mu \mathrm{g}$ of mU6pro-COX2siRNA plasmids were transfected into cells according to the manufacturer's instructions. The cells transfected with mU6pro vector alone were served as negative control. Forty-eight hours later, cells were placed in growth medium containing G418 (GIBCO) for clone selection. The expression levels of COX-2 in G418-resistant clones were evaluated by western blot analysis.

\section{RT-PCR}

All of the PCR products were separated on ethidium bromide stained agarose, and visualized with UV as described previously [6].

\section{Western blot analysis}

The western blot was done as described previously. In brief, total cellular proteins were prepared and then quantified by Bradford method [7]. A measure of 80 ug of lysates were electrophoresed in 12\% SDS-PAGE and blotted on a nitrocellulose membrane (Immoblin-P, Millipore, Bedford, MA, USA). Membranes were blocked with $5 \%$ fat-free milk powder at room temperature and incubated overnight with antibody at $4^{\circ} \mathrm{C}$. After three washes for $15 \mathrm{~min}$ in PBS-T, the membrane was incubated with the HRP-conjugated goat anti-mouse IgG antibody (Wuhan, Hubei, China) for $1 \mathrm{~h}$ at room temperature. The enhanced chemiluminescence (Amersham Life Science, Piscataway, NJ, USA) was added and monitored for the development of color.

\section{Cell growth assay}

Cells were seeded on a 96-well plate at $3 \times 10^{4}$ cells/ well. Each sample had four replicates. The medium was replaced at 2-day intervals. Viable cells were counted by the 3-[4,5-dimethylthiazol-2-yl]- 2,5-diphenyltetrazolium bromide (MTT) assay after 2, 4, 6, and 8 days.

\section{Tumor growth in nude mice}

Female athymic $n u / n u$ mice, 5-6 weeks of age, were obtained from FMMU Experimental Animal Co. (Shaanxi, China) and housed in a pathogen-free facility for all of the experiments. The logarithmically growing cells were trypsinized and resuspended in D'Hanks solution, and $5 \times 10^{6}$ cells in $0.2 \mathrm{ml}$ were injected subcutaneously into the left flank of mice [8]. Experimental and control groups had at least 6 mice each. Tumors were measured twice weekly with microcalipers, and the tumor volume was calculated according to the formula: volume $=$ length $\times\left(\right.$ width $\left.^{2}\right) / 2$.

\section{Quantification of tumor microvessel density}

Tumor microvessel densities (MVD) were quantified by anti-CD31 immunohistochemistry. Briefly, tumor sections from nude mice were cut using a LEICA cryostat and the paraffin sections were mounted on positively charged Superfrost slides and dried overnight. The immunostaining was done according to standardized protocols.

\section{Tube formation assay}

Tube formation assay was performed as described previously (Chia et al, 2010). Briefly, Confluent HUVEC cells were harvested and diluted in DMEM with 10\% FBS, which were then seeded on Matrigel-coated 24well plates. Cell culture medium was then replaced by conditioned medium. After $16 \mathrm{~h}$, Matrigel was fixed, stained with $\mathrm{H} \& \mathrm{E}$ and examined under inverted microscope. The mean tube length in five random fields per well was quantified by computer software.

\section{Cell migration assay}

Briefly, confluent monolayer of HUVEC was cultured with non-growth factor containing media for $12 \mathrm{~h}$ before harvesting. Harvested cells were suspended in serum-free DMEM199 and HUVEC cells were seeded onto tissue culture inserts in triplicate. The inserts were removed after $8 \mathrm{~h}$ culture and washed with PBS. Nonmigrated cells on the upper surface of the inserts were removed by wiping with cotton swabs. The inserts were fixed in neutral buffered formalin solution, stained with hematoxylin and eosin ( $\mathrm{H} \& \mathrm{E})$ and mounted on microscope slides. HUVEC migration was quantitated by counting the number of cells in three random fields (!200) per insert. 


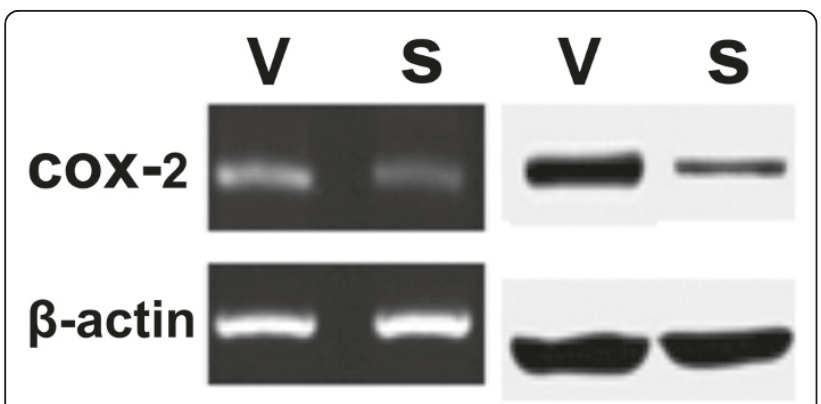

Figure 1 RT-PCR (left) and western blot analysis (right) of COX2 in the vector transfectants SGC7901-V (V) and the siRNA transfectants SGC7901-siRNA (S). B-actin was used as loading control.

\section{cDNA microarray analysis}

The gene expression was compared between SGC7901siRNA and SGC7901-vector cells for three times [9]. RNA was extracted from $80-90 \%$ confluent cells using Trizol and purified with RNeasy spin columns (Qiagen, Valencia, CA) according to the manufacturers' instructions. Quality of the RNA was ensured before labeling by analyzing 20 to $50 \mathrm{ng}$ of each sample using the RNA 6000 NanoAssay and a Bioanalyzer 2100 (Agilent, Palo Alto, CA). Samples with a peak ratio of 1.8 to 2.0 were considered suitable for labeling. Cy3- or Cy5labeled cDNA was generated and the $\mathrm{Cy} 3 / \mathrm{Cy} 5$ singlestranded cDNA/cot1 DNA pellet was resuspended in hybridization buffer, then the hybridization mix was applied to GEArray Q Series Human Angiogenesis Gene Array. The ratios of gene expression were considered to be significant if they were 2 or 0.5 in at least two independent experiments. Genes were assigned to functional families based on information from LocusLink and PubMed.

\section{Statistical analysis}

Data were presented as mean \pm standard deviation (S.D.) unless otherwise specified. Comparisons between groups were made using the Student-Newman-Keuls test or the Kruskal-Wallis test. All data were analyzed using the SPSS software package (SPSS Inc, Chicago, USA). A value of $\mathrm{P}<0.05$ was considered significant.

\section{Results}

Down-regulation of COX-2 inhibited the growth and tumorigenecity of gastric cancer cells

As Figure 1 showed, SGC7901 cells were transfected and then one resistant clone (SGC7901-siRNA) with significantly decreased COX-2 expression and one vector transfected control clone (SGC7901-vector) were selected. The results of MTT assay showed that downregulation of COX-2 might significantly decrease the proliferation of SGC7901 cells (Figure 2A). As shown in Figure $2 \mathrm{~B}$, down-regulation of COX-2 might inhibit the malignant growth of SGC7901 cells in vivo.

\section{Down-regulation of COX-2 inhibited angiogenesis of gastric cancer cells}

As shown in Figure 3, the number of endothelial cells within the tumors formed by COX-2-downregulating cells was less than that of tumors formed by control cells. In order to investigate the angiogenic property of COX-2 in endothelial cells, the in vitro tube formation of HUVEC was assessed. As shown in Figure 4, 5,

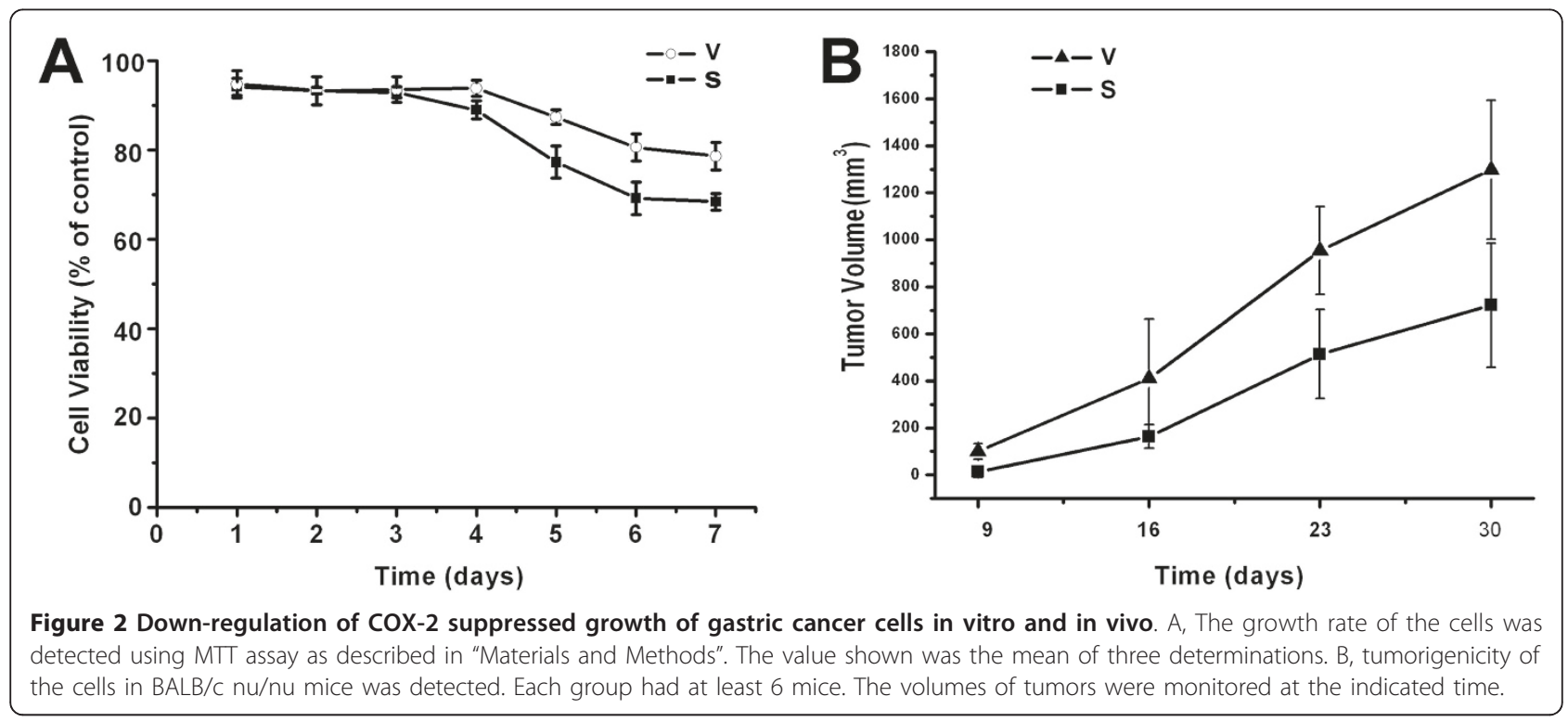




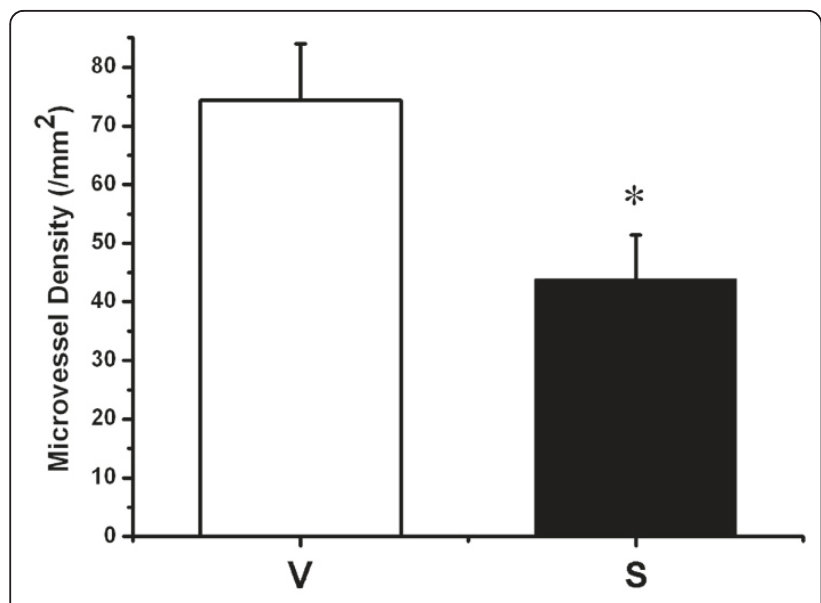

Figure 3 Effects of COX-2 on tumor angiogenesis. The tumor microvessel densities (means) in sections from tumors formed by the vector transfectants SGC7901-V ( $V$ ) and the siRNA transfectants SGC7901-siRNA (S). Tumor samples were immunostained with antibodies against CD31. Mean $\pm \mathrm{SD}, \mathrm{n}=3 .{ }^{*}, \mathrm{P}<0.05$ VS. control.

down-regulation of COX-2 might suppress cell tube formation and migration in HUVEC.

\section{Effect of COX-2 on angiogenesis related molecules}

Using cDNA microarray, genes were identified differentially expressed between different transfected SGC7901 cells. Compared with control cells, a total of 23 genes were found to be differentially expressed in COX-2downregulating cells, including FGF4, PDGF-BB, PDGFRB, PF4, TGFB2, TGFBR1, VEGF, FLT1, FLK 1, angiopoietin-1, angiopoietin-2, Tie2, IFNA1, PRL, PTN, SCYA2, SPARC, TNFSF15, PECAM1, MMP2, SERPINF1, THBS2 and OPN. To confirm the microarray findings, RT-PCR and western blot were undertaken in gastric cancer cells. Down-regulation of COX-2 might inhibit VEGF, Flt-1, Flk-1/KDR, angiopoietin-1, tie-2, MMP2 and OPN (Figure 6).

\section{Discussion}

Angiogenesis is an essential process required for the growth and metastatic ability of solid tumors. Tumor angiogenesis

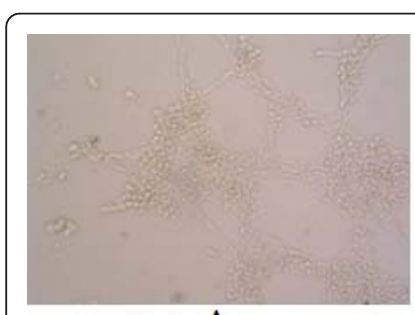

$\mathbf{A}$

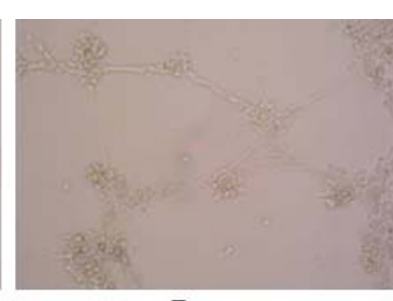

B
Figure 4 Effects of conditioned media on HUVEC tube formation. HUVECs were seeded in triplicate on Matrigelcoated 24-well plates, and incubated for $16 \mathrm{~h}$ with control SGC7901 medium (A) and COX-2-siRNA medium (B).

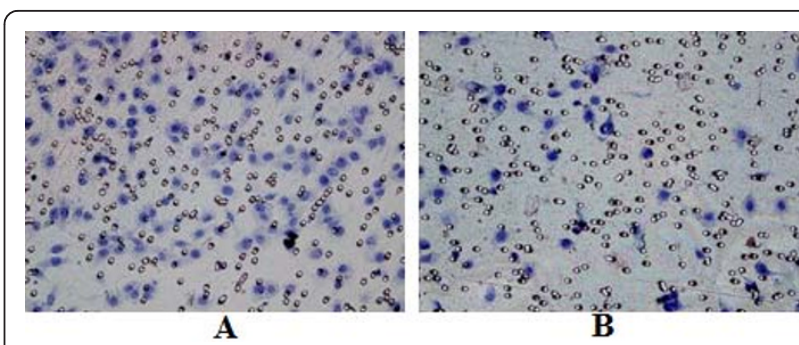

Figure 5 Effects of conditioned media on HUVEC migration. Migration assay was performed in a BioCoate Matrigele invasion chamber. The lower chambers were added with control SGC7901 medium (A) and COX-2-siRNA medium (B).

is the proliferation of a network of blood vessels penetrating into the cancerous growths to supply nutrients and oxygen and remove metabolic waste products from tumors. Tumor angiogenesis is a complex process and involves the tight interplay of tumor cells, endothelial cells, phagocytes and their secreted factors, which may act as promoters or inhibitors of angiogenesis [10]. More than a dozen different proteins (such as VEGF, bFGF, IL8, etc.), as well as several smaller molecules (such as adenosine, PGE, etc.) have been identified as angiogenic factors secreted by tumor cells to mediate angiogenesis [11,12].

Lines of evidence suggest that COX-2 is involved in the steps of gastric carcinogenesis. Increased expression

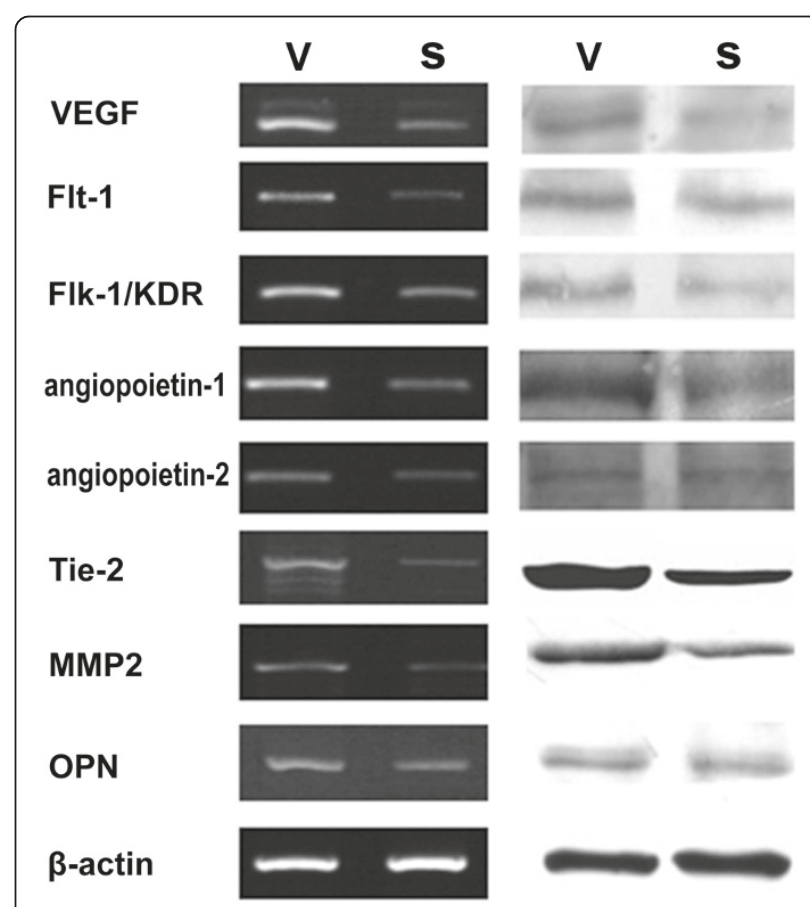

Figure 6 Expression of VEGF, Flt-1, Flk-1/KDR, angiopoietin-1, angiopoietin-2, tie-2, MMP2 and OPN in the vector transfectants SGC7901-V (V) and the siRNA transfectants SGC7901-siRNA (S) by RT-PCR (left) and Western blot (right). 
of COX-2 was frequently found in gastric cancer, in which COX-2 expression is correlated with poor prognostic outcome. Up-regulation of cox-2 expression and activity in the ulcer base not only during the acute phase of inflammation but also in the ulcer healing stage and especially in areas of intense tissue repair [13]. It has been found that cyclooxygenase- 2 inhibitors have antiproliferative and antiangiogenic activity in several types of human cancer. However, the mechanism of COX-2 in angiogenesis remains unclear.

In this study, the data showed that down-regulation of COX-2 could significantly inhibit the in vitro and in vivo growth of gastric cancer cell line SGC7901, and suppress the migration and tube formation of human umbilical vein endothelial cells, which was consistent with previous report. To our knowledge, we have firstly identified a expression pattern of angiogenesis-related molecules in COX-2mediated angiogenesis. The results of RT-PCR and western blot showed that down-regulation of COX-2 might inhibit VEGF, Flt-1, KDR, angiopoietin-1, tie-2, MMP2 and OPN.

\section{Conclusions}

In conclusion, COX-2 might mediate tumor angiogenesis and growth, and could be considered as a target for gastric cancer therapy. It was becoming increasingly clear that the signals that govern angiogenesis, functioned in complex regulatory networks rather than simple linear pathways, and that these networks might be wired differently in different cells or tumor types. The precise mechanism by which COX-2 brought about these changes, and which of these changes were primary or secondary ones, remained to be elucidated.

\section{Acknowledgement}

This study was supported in part by grants from the National Scientific Foundation of China (30873005, 30801142, 30770958 and 30871141).

\footnotetext{
Authors' contributions

Liping Yao, Fei Liu have made substantial contributions to conception and design, acquisition of data, and analysis of data. Liu Hong drafted the manuscript. Li Sun performed the statistical analysis. Shuhui Liang and Kaichun Wu have been involved in revising it critically for important intellectual content. Daiming Fan participated in its design and gave final approval of the version to be published. All authors read and approved the final manuscript.
}

\section{Competing interests}

There is no conflict of interest. The authors declare that they have no competing interests.

Received: 20 June 2010 Accepted: 25 January 2011

Published: 25 January 2011

\section{References}

1. Liu W, Zhang X, Sun W: Developments in treatment of esophageal/ gastric cancer. Curr Treat Options Oncol 2008, 9(4-6):375-87.

2. Wagner AD, Moehler M: Development of targeted therapies in advanced gastric cancer: promising exploratory steps in a new era. Curr Opin Oncol 2009, 21:381-5.
3. Wu K, Nie Y, Guo C, Chen Y, Ding J, Fan D: Molecular basis of therapeutic approaches to gastric cancer. J Gastroenterol Hepatol 2009, 24(1):37-41.

4. Mrena J, Wiksten JP, Kokkola A, Nordling S, Ristimäki A, Haglund C: COX-2 is associated with proliferation and apoptosis markers and serves as an independent prognostic factor in gastric cancer. Tumour Biol 2010, 31(1):1-7.

5. Hong L, Zhao Y, Han Y, Guo W, Jin H, Qiao T, Che Z, Fan D: Mechanisms of growth arrest by zinc ribbon domain-containing 1 in gastric cancer cells. Carcinogenesis 2007, 28(8):1622-8.

6. Hong L, Wang J, Zhao Y, Han Z, Zhou X, Guo W, Zhang X, Jin H, Wu K, Ding J, Fan D: DARPP-32 mediates multidrug resistance of gastric cancer through regulation of P-gp and ZNRD1. Cancer Invest 2007, 25(8):699-705.

7. Han Z, Hong L, Han Y, Wu K, Han S, Shen H, Li C, Yao L, Qiao T, Fan D: Phospho Akt mediates multidrug resistance of gastric cancer cells through regulation of P-gp, Bcl-2 and Bax. J Exp Clin Cancer Res 2007, 26(2):261-8.

8. Hong L, Piao Y, Han Y, Wang J, Zhang X, Du Y, Cao S, Qiao T, Chen Z, Fan D: Zinc ribbon domain-containing 1 (ZNRD1) mediates multidrug resistance of leukemia cells through regulation of P-glycoprotein and Bcl-2. Mol Cancer Ther 2005, 4(12):1936-42

9. Hong L, Han Y, Zhang H, Li M, Gong T, Sun L, Wu K, Zhao Q, Fan D: The prognostic and chemotherapeutic value of miR-296 in esophageal squamous cell carcinoma. Ann Surg 2010, 251(6):1056-63.

10. Chia JS, Du JL, Hsu WB, Sun A, Chiang CP, Wang WB: Inhibition of metastasis, angiogenesis, and tumor growth by Chinese herbal cocktail Tien-Hsien Liquid. BMC Cancer 2010, 10(1):175.

11. Cao $Y$ : Adipose tissue angiogenesis as a therapeutic target for obesity and metabolic diseases. Nat Rev Drug Discov 2010, 9(2):107-15.

12. Elewa HF, El-Remessy AB, Somanath PR, Fagan SC: Diverse effects of statins on angiogenesis: new therapeutic avenues. Pharmacotherapy 2010, 30(2):169-76.

13. Na rdone $\mathrm{G}$, Rocco A: Chemoprevention of gastric cancer: role of COX-2 inhibitors and other agents. Dig Dis 2004, 22(4):320-6.

doi:10.1186/1756-9966-30-13

Cite this article as: Yao et al:: The function and mechanism of COX-2 in angiogenesis of gastric cancer cells. Journal of Experimental \& Clinical Cancer Research 2011 30:13.

\section{Submit your next manuscript to BioMed Central and take full advantage of:}

- Convenient online submission

- Thorough peer review

- No space constraints or color figure charges

- Immediate publication on acceptance

- Inclusion in PubMed, CAS, Scopus and Google Scholar

- Research which is freely available for redistribution 